PGA and physician global health assessments were compared using Wilcoxon rank-sum tests.

Results: 16 parents and 31 patients completed the assessments (table 1). There was a moderate correlation between PGA and JADAS-71 ( $r=0.503, p<0.001)$, and $P G A$ and physician global health assessments $(r=0.503, p=0.002)$. There was a stronger correlation between physician global health assessments and JADAS71. PGA median scores and IQRs appeared to be higher among patients with Medicaid insurance, non-white race, and Hispanic ethnicity, with the greatest difference seen in the category of race (Table 2). There were no differences between patient and physician assessments across all groups except among patients with Medicaid (difference median $=-1.25$ ) and Hispanic patients (difference median=2)

Abstract AB1377 - Table 1. Patient Characteristics

\begin{tabular}{lccc}
\hline & $\begin{array}{c}\text { Patients and Parents } \\
(\mathbf{n}=\mathbf{4 7})\end{array}$ & $\begin{array}{c}\text { Patients } \\
(\mathbf{n}=31)\end{array}$ & $\begin{array}{c}\text { Parents } \\
(\mathbf{n}=16)\end{array}$ \\
\hline $\begin{array}{l}\text { Age, years } \\
\text { (median, IQ range) }\end{array}$ & 12.4 & 14.2 & 4.3 \\
Male Gender & {$[8.6-15.0]$} & {$[12.4-15.8]$} & {$[3.3-9.0]$} \\
Race & $17(36.2 \%)$ & $14(45.2 \%)$ & $3(18.8 \%)$ \\
Caucasian/White & & & \\
Asian & $38(80.9 \%)$ & $24(77.4 \%)$ & $14(87.5 \%)$ \\
Other & $3(6.4 \%)$ & $3(9.7 \%)$ & $0(0.0 \%)$ \\
Hispanic Ethnicity & $6(12.7 \%)$ & $4(12.9 \%)$ & $2(12.5 \%)$ \\
Insurance & $8(17.0 \%)$ & $5(16.1 \%)$ & $3(18.8 \%)$ \\
Medicaid & & & \\
Private & $8(17.0 \%)$ & $4(12.9 \%)$ & $4(25.0 \%)$ \\
& $39(83.0 \%)$ & $27(87.1 \%)$ & $12(75.0 \%)$ \\
\hline
\end{tabular}

Abstract AB1377 - Table 2. Comparison of Patient Global Assessments by age, sex, insurance status, race and ethnicity

\begin{tabular}{lcr}
\hline & Patient global median [IQR] & P-value \\
\hline Age & $2[0-5]$ & 0.892 \\
Patient completed & $2[0.05-4.5]$ & \\
Parent completed & $2[0-5]$ & 0.679 \\
Sex & $2[0-4]$ & \\
Male & & \\
Female & $2.5[1,5]$, & 0.313 \\
Insurance & $2[0-4]$ & \\
Medicaid & & 0.266 \\
Private & $1.5[0-4]$ & \\
Race & $4\left[{ }^{2-5}\right]$ & \\
White & $2.5[2.5-5]$ & 0.134 \\
Non-white & $1[0-4]$ & \\
Ethnicity & & \\
Hispanic & Non-Hispanic &
\end{tabular}

Conclusions: Our results demonstrate that physician global health assessment had a stronger correlation with standard disease activity measures than the PGA. These scores were higher in patients who were non-White race, Hispanic, and had Medicaid insurance; however, these were not statistically significant. These data indicate that the PGA is fairly stable across groups, and can be used reliably for disease monitoring.

Disclosure of Interest: None declared

DOI: 10.1136/annrheumdis-2018-eular.7351

\section{AB1378 CLINICAL COMPARISON OF NEW CRITERIA FOR INFLAMMATORY MYOPATHY IN A COLOMBIAN COHORT}

R. Fuentes ${ }^{1}$, D. Gil-Calderon ${ }^{1}$, M. Cañola ${ }^{1}$, J.C. Diaz-Coronado ${ }^{2}$, A. RojasVillarraga $^{2}$, D. Hernandez-Parra ${ }^{2}$, P. Perez-Estrada ${ }^{2}$, J.C. Salazar-Uribe ${ }^{3}$, R. Pineda-Tamayo ${ }^{2} .{ }^{1}$ Rheumatology; ${ }^{2}$ Clinical Research, Artmedica; ${ }^{3}$ National University of Colombia, Medellin, Colombia

Background: Idiopathic inflammatory Myopathies are a group of diseases characterised by weakness due to muscle inflammation, with or without dermatological involvement, that classically includes pathognomonic findings like Gottron sign. There have been different criteria used to classify these diseases, being Peter and Bohan the most used in time, but recently the ARC/EULAR group proposed a new wat to approach the classification.

Objectives: To compare ACR/EULAR 2017 versus Peter and Bohan criteria for Idiopathic Inflammatory Myopathy in a Colombian cohort

Methods: A cross-section retrospective research was done with data collected between 2014 and 2017 from a population diagnosed with Idiopathic Myopathy according to Peter and Bohan criteria and followed up for at least six months. The new ACR/EULAR criteria were applied to each individual using the online tool (hhtp://www.imm.ki.se/biosatistics/calculators/iim). Both sets of criteria were compared using Cohen's kappa coefficient and concordance was evaluated.

Results: Data of 149 patients were obtained. Anti-Jo1 results were not available for $75 \%$ of the patients. Biopsy was available in $44,3 \%$ of patients. Biopsy results were compatible with inflammatory myopathy in $66,7 \%$ and non-compatible in $33,3 \%$. According to Peter and Bohan criteria the diagnosis of idiopathic inflammatory myopathy was definite in $63,1 \%$ of the patients, and probable in $27,5 \%$. Using ACR/EULAR 2017 criteria instead the diagnosis was definite in 63,1\%, probable $10,1 \%$ and non-possible in 20,8\%. According to the new criteria, 31 patients had polymyositis, 47 dematomyositis, 4 amyopathic dermatomyositis, 35 juvenile myositis and 1 inclusion body myositis. The concordance analysis between the two sets of criteria showed agreement of $54 \%$ (kappa $0,22 p<0,001$ ) in the whole group, 59\% (kappa 0,21 $\mathrm{p}<0,001$ ) in adults, 32\% (kappa $0,18 \mathrm{p}=0,05$ ) in children, $44 \%$ (kappa $0,05 \mathrm{p}=0,3$ ) in polymyositis and $42 \%$ (kappa $0,16 \mathrm{p}=0,1$ ) in dermatomyositis.

Conclusions: Data of 149 patients were obtained. Anti-Jo1 results were not available for $75 \%$ of the patients. Biopsy was available in $44,3 \%$ of patients. Biopsy results were compatible with inflammatory myopathy in $66,7 \%$ and noncompatible in $33,3 \%$. According to Peter and Bohan criteria the diagnosis of idiopathic inflammatory myopathy was definite in $63,1 \%$ of the patients, and probable in $27,5 \%$. Using ACR/EULAR 2017 criteria instead the diagnosis was definite in $63,1 \%$, probable $10,1 \%$ and non-possible in $20,8 \%$. According to the new criteria, 31 patients had polymyositis, 47 dematomyositis, 4 amyopathic dermatomyositis 35 juvenile myositis and 1 inclusion body myositis. The concordance analysis between the two sets of criteria showed agreement of 54\% (kappa 0,22 $p<0,001$ ) in the whole group, 59\% (kappa 0,21 p<0,001) in adults, 32\% (kappa 0,18 $\mathrm{p}=0,05$ ) in children, $44 \%$ (kappa $0,05 \mathrm{p}=0,3$ ) in polymyositis and $42 \%$ (kappa 0,16 $\mathrm{p}=0,1)$ in dermatomyositis.

\section{REFERENCE:}

[1] I. Lundberg, et al. 2017 European League Against Rheumatism/American College of Rheumatology classification criteria for adult and juvenile idiopathic inflammatory myopathies and their major subgroups. Ann Rheum Dis 2017:0:1-10. doi:10.1136/annrheumdis-2017-211468

Disclosure of Interest: None declared

DOI: 10.1136/annrheumdis-2018-eular.2189

\section{AB1379 POTENTIAL DIAGNOSTIC SERUM IMMUNOLOGICAL MARKER PANEL IN PRIMARY AND SECONDARY OSTEOARTHRITIS IN SRI LANKAN PATIENTS}

S. Rankotgedera ${ }^{1}$, I. Atukorala ${ }^{2}$, D. Munidasa ${ }^{3}$, L. Wijayaratne ${ }^{4}$, P. Udagama ${ }^{1}$. ${ }^{1}$ Department of Zoology and Environment Sciences, University of Colombo, Sri Lanka, Colombo 3; ${ }^{2}$ Department of Clinical Medicine, University of Colombo, Sri Lanka, Colombo $8 ;{ }^{3}$ Rheumatology and Rehabilitation Hospital, Ragama; ${ }^{4}$ Department of Zoology and Environment Sciences, Nawaloka Hospital PLC, Colombo 2, Sri Lanka

Background: Osteoarthritis $(O A)$ is commonly perceived as a degenerative joint disease but it is now established that inflammation contributes to OA. ThoughOA is categorised into primary and secondary osteoarthritis, most clinicians manage patients of both categories in a similar manner.

Objectives: The current study aimed to identify potential diagnostic markers of primary and secondary OA. Two cohorts of Sri Lankan patients, one with primary $\mathrm{OA}$ and another with secondary $\mathrm{OA}$ were assessed for a selected panel of immunologic mediators i.e. cytokines (TNF- $\alpha, \mathrm{IL}-1 \beta, \mathrm{IL}-6, \mathrm{IL}-10)$ and nitric oxide derivatives (NOx).

Methods: A case control study was conducted with 40 OA patients (Primary, $\mathrm{n}=30$; Secondary, $\mathrm{n}=10$ ), and 60 age and gender matched controls (normal healthy, $n=30$; Systemic Lupus Erythematosus (SLE) disease control, $n=30$ ). The socioeconomic and demographic data were accrued via an interviewer adminis tered questionnaire. Sandwich ELISAs assayed serum cytokine levels, while the 'Griess assay' measured serum NOx levels.

Results: In comparison to healthy controls, OA patients showed significantly higher serum concentrations of all five analytes tested $(p<0.05)$. Patients with primary OA had significantly higher levels of TNF- $\alpha$ and IL-1 $\beta$ and lower level of IL- 6 in serum compared to disease controls (SLE) $(p<0.05)$. secondary OA patients exhibited a significant increase in serum TNF- $\alpha$ and IL-1 $\beta$ and lower serum IL-10 levels compared to disease controls $(p<0.05)$. In the two test groups, the serum levels of TNF- $\alpha$ and IL- 6 were significantly elevated in secondary OA patients $(p<0.05)$. The NOx concentrations between the two test groups was not significantly different. The Th1:Th2 cytokine ratio (TNF- $\alpha: I L-10)$ was significantly higher in secondary OA compared with primary $O A(p<0.05)$.

The Receiver operating characteristic (ROC) curves identified TNF- $\alpha$ and IL-10 as potential diagnostic biomarkers of both primary $\mathrm{OA}$ and secondary $\mathrm{OA}$ compared with healthy controls. IL1- $\beta$ and IL- 6 may be used specifically as diagnostic 
biomarkers for primary and secondary OA, respectively, when compared with healthy individuals. TNF- $\alpha$ is a suitable biomarker for the diagnosis of both primary and secondary OA when compared to SLE patients, whereas IL- 6 and IL-1 $\beta$ were apparent explicit markers of primary $\mathrm{OA}$, while $\mathrm{IL}-10$ was exclusive for secondary OA.

Abstract AB1379 - Table 1. lists the values obtained from the ROC curves for the cytokine panel and NOx for patients clinically diagnosed with primary and secondary osteoarthritis against normal healthy controls.

\begin{tabular}{|c|c|c|c|c|c|c|c|c|}
\hline & \multicolumn{4}{|c|}{ Primary $\mathrm{OA}$} & \multicolumn{4}{|c|}{ Secondary $\mathrm{OA}$} \\
\hline & $\begin{array}{l}\text { AUC } \\
(\%)\end{array}$ & $\begin{array}{l}\text { Cut-off } \\
\text { value }^{*}\end{array}$ & Sensitivity & Specificity & $\begin{array}{c}\text { AUC } \\
(\%)\end{array}$ & $\begin{array}{l}\text { Cut-off } \\
\text { value }^{*}\end{array}$ & Sensitivity & Specificity \\
\hline $\begin{array}{l}\text { TNF- } \\
\alpha\end{array}$ & 88.3 & $>10.8$ & 93.33 & 80.00 & 97.0 & $>28.2$ & 100.00 & 96.67 \\
\hline IL-10 & 88.6 & $>32.67$ & 85.71 & 92.59 & 93.1 & $>32.67$ & 90.00 & 92.59 \\
\hline IL-6 & 59.3 & $>3.98$ & 89.66 & 40.74 & 81.2 & $>12.02$ & 66.67 & 96.15 \\
\hline IL-1 $1 \beta$ & 85.2 & $>1.18$ & 96.43 & 68.42 & 61.4 & $>0.88$ & 77.78 & 57.89 \\
\hline NOx & 72.0 & $>18.89$ & 93.33 & 60.71 & 73.0 & $>18.89$ & 100.00 & 60.71 \\
\hline
\end{tabular}

${ }^{\star}$ For the cytokines, in $\mathrm{pg} / \mathrm{ml}$ and for $\mathrm{NOx}$ in $\mu \mathrm{mol} / \mathrm{l}$

Conclusions: This preliminary study suggests that higher levels of inflammatory cytokines are present in secondary OA compared to primary OA. Furthermore, distinct markers for primary and secondary $O A$ were identified, indivative of the potential for developing different therapeutic agents for the different types of OA. Acknowledgements: The authors acknowledge the University of Colombo, Sri Lanka for funding.

Disclosure of Interest: None declared

DOI: 10.1136/annrheumdis-2018-eular.6602

\section{AB1380 PERIPHERAL NEUROPATHY IN INFLAMMATORY JOINT DISEASES}

Y. Yehudina ${ }^{1}$, O. Syniachenko ${ }^{2}$, T. Bevzenko ${ }^{3} .{ }^{1}$ internal Medicine, Dnepropetrovsk Medical Academy, Dnipro; ${ }^{2}$ Donetsk Medical University, Lyman; ${ }^{3}$ Shupyk National Medical academy, Kyiv, Ukraine

Background: For frequent extra-articular (systemic) manifestations of joints inflammatory diseases are various damage of the nervous system, ${ }^{2}$ and the presence and severity of peripheral neuropathy (PNP) have significance in the clinical course, which incidence among these patients is $5 \%-10 \% .{ }^{1}$ Nevertheless, many clinical and pathogenic aspects of this peripheral neuropathy (PNP) remain obscure.

Objectives: to evaluate the rate and clinical features of PNP in rheumatoid arthritis (RA), chlamydia urogenital reactive arthritis (ReA), psoriatic arthritis $(P A)$ and ankylosing spondylitis (AS), to examine the issues of pathogenetic constructions such changes of the nervous system, define risk factors.

Methods: The study included 416 patients with inflammatory joint diseases, among them 131 RA patients, 101 ReA, 76 PA and 108 AS. The average age of the examined was respectively $45,32,42$ and 38 years, disease duration $-10,4$, 12 and 11 years, male to female ratio $-1: 5,1: 1,1: 2$ and $10: 1$

Results: The frequency of the PNP in RA, ReA, PA and AS was 13\%, 19\%, 24\% and $34 \%$, while its severity in patients with $P A<R e A<R A<A S$, and the same type ratio of motor, sensory and mixed disorders of inflammatory joints diseases, trophic, visceral and vascular vegetative changes, the connexion with the male sex, the activity of arthritis and the presence of tendovaginitis, participation of immune disorders, endothelial dysfunction of blood vessels and changes in physical and chemical rheological viscoelastic properties of blood in the pathogenesis constructions of the nervous system lesions are united around. ReA and PA are different by frequency of hands and feet distal pathology, the AS - by the beginnings of tunnel syndrome. RA tends to impact on the PNP digital arteritis, myositis, eye disease, and Sjogren's syndrome, ReA - on sacroilitit, PA - on exudative form of cutaneous psoriasis, AS - on eye disease, at that, the risk factors for severe course of neuropathy in RA is considered to involve in the process of elbows, $R e A$ - intervertebral and facet joints, PA - wrist, AS - sacroiliac. Guillain-Barré syndrome develops respectively in $3 \%, 4 \%, 5 \%$ and $9 \%$ of patients with RA, PA, ReA and AS, or in $24 \%, 17 \%, 26 \%$ and $27 \%$ cases of PNP, which is closely linked to the presence of tendovaginitis in all inflammatory diseases and severity of articular syndrome, in RA it depends on the presence of hypothyroidism, in ReA - on nephropathy and violations of the heart's electrical conduction, in AS - on osteoporosis, and seropositive for anti-cyclic citrullinated peptide antibody is a risk factor for such peripheral nervous system disorders.

Conclusions: PNP is a relatively common manifestation of inflammatory diseases of the joints, which correlate with clinical and laboratory signs of the disease, and in the future such active detection of the nervous system pathology will be useful for timely follow-up rehabilitation.

\section{REFERENCE:}

[1] Oomatia A., Fang H., Petri M., Birnbaum J. Peripheral neuropathies in systemic lupus erythematosus: clinical features, disease associations, and immunologic characteristics evaluated over a twenty-five-year study period. Arthritis Rheumatol 2014;66(4):1000-1009.

[2] Sampaio-Barros PD. Epidemiology of spondyloarthritis in Brazil. Am. J. Med. Sci 2011;341(4): 287-288.

Disclosure of Interest: None declared

DOI: 10.1136/annrheumdis-2018-eular.3736

\section{Education}

\section{AB1381 FOUR ANNUAL INTERNATIONAL DIFFERENT MEETINGS OF RHEUMATOLOGY: COMPARATION OF THE CONTENTS, ANALYSIS, CHALLENGE AND OPORTUNITIES}

A.J. Pedro ${ }^{1}$, E. Cuevas ${ }^{1}$, C. Abud ${ }^{1}$, R. Moreno ${ }^{1}$, M. Martinez ${ }^{1}$, D. Herrera ${ }^{1}$ E. Acevedo ${ }^{2}$, H. Lopez ${ }^{2}$, D. Ramos ${ }^{2}$, G. Aguilera Barragan², G. Martinez ${ }^{2}$,

T. Luna ${ }^{2}$, E. Santillan ${ }^{2} .{ }^{1}$ Rheumatology; ${ }^{2}$ Hospital Central Ignacio Morones Prieto, San Luis Potosí, Mexico

Background: The medical meetings are a tool to help us be able to escalate and actualize the medical knowledge and their quality is a responsibility of Colleges and Institutions.

Objectives: To assess the academic level of four types of different annual Meeting of Rheumatology

Methods: We used as support information the summaries published in the sup plements of the journal Reumatologia Clinica, SE1 Vol. 12 of February 2016, the supplement SE 1 Vol. 13 of February 2017, the application for electronic media of the ACR/ARHP 2016 of the American Congress of Rheumatology 2016 and the website for abstracts of ,EULAR 2017 dedicated to the surveys presented in the XLIV Mexican Congress of Rheumatology, XLV Mexican Congress of Rheumatology and the 2016 ACR/ARHP Annual Meeting, and EULAR 2017 respectively, of each survey we was obtained information about of the diverse pathologies, the type of trial, content and population referred (adults versus children).

Results: $275,340,3275$ and 4129 were presented in the XLIV Mexican Congress of Rheumatology, XLV Mexican Congress of Rheumatology, the 2016 ACR/ARHP Annual Meeting and EULAR 2017 respectively. Rheumatoid arthritis (RA) was the most common pathology with 23\%, 26\%, 21\% and 27\% in CMR 44, CMR 45, ACR 2016 and EULAR 2017 respectively, followed by systemic lupus erythematosus, third place was vasculitis, beside in international congress was the spondyloartrhitis Highlighted, RA the items about of clinic manifestations were accounted for almost $30 \%$ in the Mexican congress and almost $20 \%$ in ACR and EULAR.

Observational studies accounted for almost $40 \%$ in Mexican congresses vs. $33 \%$ in ACR 2016 and $55 \%$ in, EULAR 2017 beside surveys about of basic research were minimal in Mexican congress, but in ACR 2016 accounted for $21 \%$ and $12 \%$ in EULAR 2016

The trials about of Paediatric Rheumatology were $12.3 \%, 5.5 \%$ and $4.9 \%$ in CMR 44, CMR 45 and ${ }^{\text {ACR } 2016}$ respectively.

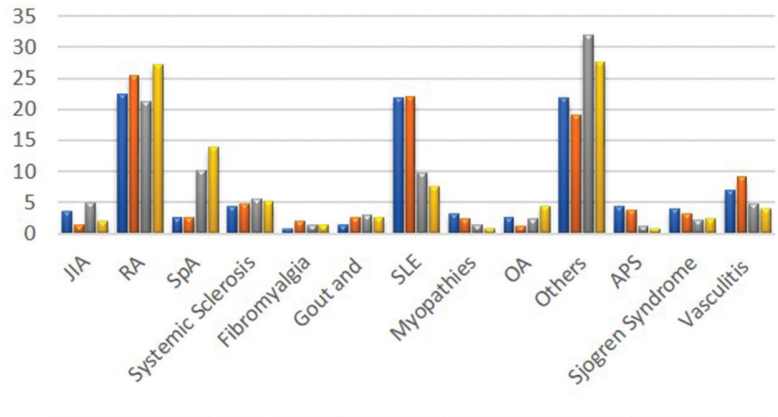

घCMR 44 घCMR 45 घ2016 ACR/ARHP Annual Meeting घEULAR 2017

Abstract AB1381 - Figure 1. percentage of rheumatic disease

Conclusions: Rheumatology Meeting constitutes a support to obtain the adequate medical knowledge based in evidence, in this important branch of 